\title{
APPROXIMABILITY OF THE INVERSE OF AN OPERATOR
}

\author{
AVRAHAM FEINTUCH
}

\begin{abstract}
Let $A$ be an invertible operator on a complex Hilbert space $\mathcal{X}$. A necessary and sufficient condition is given for $A^{-1}$ to be a weak limit of polynomials in $A$.
\end{abstract}

Let $A$ be a bounded linear invertible operator on a complex Hilbert space $\mathcal{H}$. If $\mathcal{H}$ is finite dimensional then there exists a polynomial $P$ such that $A^{-1}=p(A)$. The expected infinite dimensional analogue is false. If $\mathcal{H}$ is infinite dimensional then $A^{-1}$ is not in general a limit of polynomials in $A$ even in the weak operator topology; a simple example is the bilateral shift. Sufficient conditions for $A^{-1}$ to be a weak (equivalently, strong) limit of polynomials in $A$ were given in [2] and [3]. Here a necessary and sufficient condition is presented in the spirit of Theorem 1 of [2]. The proof was motivated by some results given in [1].

Let $\mathcal{R}$ denote the weak closure of the algebra of polynomials in $A, \mathcal{F}^{(n)}$ the usual direct sum of $n$ copies of $\mathcal{H}, A^{(n)}$ the operator on $\mathcal{H}^{(n)}$ defined by

$$
A^{(n)}\left\langle x_{1}, \ldots, x_{n}\right\rangle=\left\langle A x_{1}, \ldots, A x_{n}\right\rangle
$$

and $\mathscr{R}^{(n)}=\left\{T^{(n)}: T \in \Re\right\}$. Lat $A$ will denote the lattice of closed invariant subspaces of $A$. The characterization of $\Re$ given in Lemma 1 is standard [5, p. 118].

LEMMA 1. $T \in \mathscr{R}$ if and only if Lat $A^{(n)} \subseteq$ Lat $T^{(n)}$ for all $n \geqslant 1$.

- Definition. The operator $T$ is strictly positive if there exists a real number $\delta>0$ such that for all $f \in \mathcal{H}, \operatorname{Re}(T f, f) \geqslant \delta\|f\|^{2}$.

LEMMA 2. If $T$ is strictly positive, so is $T^{(n)}$ for $n \geqslant 1$.

Proof. Left to the reader.

THEOREM. Let $A$ be an invertible operator on $\mathcal{H}, \mathcal{R}$ the weak closure of the algebra of polynomials in $A$. Then $A^{-1} \in \Re$ if and only if there exists $T \in \Re$ such that $T^{-1} \in \mathcal{R}$ and $T A$ is strictly positive.

Proof. If $A^{-1} \in \mathscr{R}$ just pick $T=A^{-1}$. Suppose $T, T^{-1} \in \mathscr{R}$ and $T A$ is strictly positive. We show Lat $A \subset$ Lat $A^{-1}$. Let $\Re \in$ Lat $A$ and $P$ be the

Received by the editors March 4, 1977.

AMS (MOS) subject classifications (1970). Primary 47C05.

$\mathrm{Key}$ words and phrases. Invertible operator, weakly closed algebra, strict positivity, numerical range. 
orthogonal projection on $\Re$. We will denote by $P S P$ the operator $P S \mid \mathfrak{T}$. Then to show $\Re \in$ Lat $A^{-1}$, it suffices to show that $P A P$ is invertible for if this is the case, $\Re=P A P \Re=A \Re$ (since $\Re \in$ Lat $A$ ) and thus $A^{-1} \Re$ $=\Re$. Since $T, T^{-1} \in \Re, P T P$ is invertible with inverse $P T^{-1} P$. Thus the invertibility of $P A P$ is equivalent to the invertibility of $P T A P=P T P A P$. We show PTAP is invertible.

By our assumption that $T A$ is strictly positive, there is some $\delta>0$ such that $\operatorname{Re}(T A f, f) \geqslant \delta\|f\|^{2}$ for all $f$ in $\mathcal{H}$. Applied in particular to $f$ in $\mathscr{N}$ this shows that PTAP is strictly positive and thus invertible. Therefore Lat $A \subset$ Lat $A^{-1}$.

We complete the proof by noting that the same argument applies to show Lat $A^{(n)} \subseteq$ Lat $A^{-1(n)}$ and applying Lemma 1 .

Let $\omega(T)$ denote the numerical range of $T=\{(T x, x):\|x\|=1\}$.

CoRollary. $A^{-1} \in \Re$ if and only if there exists $T \in \Re$ such that $T^{-1} \in$ $\Re$ and $0 \notin \omega(T A)$.

\section{BiBLIOGRAPHY}

1. A Devinatz and M. Shinbrot, General Wiener-Hopf operators, Trans. Amer. Math. Soc. 145 (1969), 467-494.

2. A. Feintuch, On invertible operators and invariant subspaces, Proc. Amer. Math. Soc. 45 (1974), 123-126.

3. __ Algebras generated by invertible operators, Proc. Amer. Math. Soc. 63 (1977), 66-68.

4. R. G. Douglas, Banach algebra techniques in operator theory, Academic Press, New York and London, 1972.

5. H. Radjavi and P. Rosenthal, Invariant subspaces, Springer-Verlag, Berlin, Heidelberg, New York, 1973.

Department of Mathematics, Ben Gurion University of Negev, Beer Sheva, Israzl 